\title{
LA REDEFINICIÓN DEL ESPACIO PÚBLICO: EL CASO DE LASESCUELAS AUTOGESTIONADAS EN ARGENTINA
}

\author{
MYRIAM FELDFEBER*
}

\begin{abstract}
Esta nobleza de Estado, que predica la extinción del Estado y el reinado conjunto del mercado y el consumidor, sustituto comercial del ciudadano, se ha apoderado del Estado; ha convertido el bien público en bien privado, la cosa pública, la República en su cosa. Lo que está en juego actualmente, es la reconquista de la democracia contra la tecnocracia.
\end{abstract}

(Bourdieu, Contrafuegos)

RE SU ME N : El Proyecto de las Escuelas Experimentales o Autogestionadas de la Provincia de San Luis adquiere sentido en el marco de la denominada Transformación Educativa y de las políticas desarrolladas en las últimas décadas en Argentina, que, al igual que en gran parte de los países latinoamericanos, han estado orientadas por discursos y prácticas tendientes a legitimar un nuevo modelo económico, social, político, cultural y educativo. La reforma educativa argentina de la década del ' 90 puede analizarse como una respuesta frente a la crisis de la matriz estado-céntrica que caracterizó la institucionalización y expansión del sistema educativo. El Proyecto de las Escuelas Experimentales en la Provincia de San Luis, inspirado en el modelo de las charter schools de Estados Unidos, es presentado por el Gobierno de la Provincia como un caso precursor en nivel regional en el marco de lo que caracterizan como la "necesaria e imperante reforma de los sistemas educativos". En este sentido, el objetivo de este trabajo es analizar la lógica que orienta el modelo de estas escuelas así como presentar algunas problemáticas centrales vinculadas con el proceso de implementación de la propuesta.

Palabras daves: Escuelas autogestionadas. Organizaciones públicas noestatales. Casi-mercados. Escuela pública. Reforma educativa.

Profesora e investigadora de la Universidad de Buenos Aires. E-mail: mfeldfeb@ filo.uba.ar/ correo@myriamf.com 


\section{REDEFINING THE PUBLIC SPHERE: Self-Managed schools in Argentina}

A BSTRA CT: The educational reform that took place in Argentina during the '90s was an answer to the crisis of the state-centered model that fostered the expansion of the school system in the XX ${ }^{\text {th }}$. Century. This reform assumed that the solution to improve the quality of education should focus on such issues as: reducing the role of the state in the system assessment; profoundly reforming the school organization; considering new forms of demand-side financing; introducing incentives; giving autonomy to individual schools; involving non-state institutions in policy management; producing information in order to guarantee the freedom of choice; offering new career for teachers based on merit and derogation of the labor statute. Nevertheless, the educational reform should be analyzed in the context of a broader reform process implemented in the whole Latin America in the '90s, aimed at legitimating a new economic, social, political and cultural model. Inspired in the "charter schools" model, the Experimental Schools Project in the Province of San Luis is a leading case in the region of what is characterized as the "necessary and imperative reform of the educational systems". In this context, the aim of this article is both to analyze the logic behind this model and to discuss the main issues of its implementation process.

Key words: Charter schools. Non state public organizations. Quasimarkets. Public school. Educational reform.

\section{Introducción}

$E$ n los últimos años, tanto en el nivel del discurso de los expertos ministeriales como de los técnicos del Banco Mundial y de las fundaciones locales de orientación neoliberal, se han instalado los temas de la autonomía institucional, los nuevos modelos de gestión y la redefinición de la carrera docente como aspectos centrales del proceso de reforma pendiente en el campo educativo en Argentina. Para los tecnócratas locales la solución para elevar la calidad del sistema educativo debería estar centrada en las siguientes medidas: una redefinición del papel del Estado centrado en la evaluación del sistema; una reforma profunda en la organización escolar; el establecimiento de nuevas formas de financiamiento educativo basadas en la demanda; la introducción de incentivos; el otorgamiento de autonomía a la escuela transformándola en protagonista del sistema; la implementación de políticas públicas gestionadas por organizaciones no estatales; la producción de información para garantizar la libertad 
de elección a los padres; la definición de una nueva carrera basada en el mérito y la modificación del régimen laboral docente. ${ }^{1}$

En el año 2001 comenzaron a funcionar en la Provincia de San Luis las "Escuelas Experimentales o Escuelas 2001" - también denominadas "Escuelas Autogestionadas" - que constituyen una propuesta que articula los aspectos centrales de estas reformas impulsadas por los "expertos". D e acuerdo con las propias autoridades de la Provincia estas escuelas "encuentran sus dinámicos pero escasos antecedentes sólo en reformas muy recientes, realizadas en distintos grados y desde 1991 por más de treinta estados de los Estados Unidos y, en menor grado de profundidad, por municipios de Chile, y por algunos países europeos. Son pocos aún, sin embargo, quienes se han decidido avanzar en la modernización de los sistemas educativos con la profundidad con que nuestra provincia se lo ha propuesto. Esto hace de San Luis un caso único en nuestro país, que será analizado de cerca por las demás provincias argentinas e incluso por otros países de la región".

El Proyecto de las Escuelas Experimentales en la Provincia de San Luis es presentado por el Gobierno de la Provincia como un caso precursor en nivel regional en el marco de lo que caracterizan como la "necesaria e imperante reforma de los sistemas educativos". En este sentido, el objetivo de este trabajo ${ }^{3}$ es analizar la lógica que orienta el modelo de estas escuelas así como presentar algunas problemáticas centrales vinculadas con el proceso de implementación, con las limitaciones derivadas del hecho de investigar sobre un proceso en curso. ${ }^{4}$

\section{Los casi-mercados en educación como espacios públicos no-estatales}

Las Escuelas Experimentales de San Luis son caracterizadas desde la retórica oficial como organizaciones públicas no-estatales. Estas organizaciones surgen como respuesta a la crisis del modelo social burocrático del Estado y a los requerimientos de una administración pública más eficiente en el contexto de la globalización (Bresser Pereira, 1998). Son organizaciones que se "localizan" entre el Estado y el mercado.

Las organizaciones públicas no-estatales, de acuerdo con la definición de Bresser Pereira y Cunill Grau (1998), constituyen organizaciones o formas de control públicas porque están volcadas al interés general y son no-estatales porque no forman parte del aparato del Estado, sea porque no utilizan servidores públicos o porque no coinciden con los agentes políticos tradicionales. Entre las ventajas de estas 
organizaciones señalan: a) pluralización de la oferta de los servicios por la oportunidad de desarrollar el principio de competencia, lo que posibilita que los usuarios ejerciten el derecho a la salida, lo que estimula la calidad y la eficiencia en la prestación del servicio; b) flexibilización y desburocratización de la gestión social, posibilitando obtener prestaciones adaptadas a los públicos concernidos, en oposición a las prestaciones uniformes que tienden a caracterizar la oferta pública estatal; c) responsabilidad de los dirigentes y participantes de la organización que deben utilizar adecuadamente los recursos para lograr los objetivos propuestos.

D e este modo las organizaciones públicas no-estatales asumen el modelo de funcionamiento de los denominados casi-mercados que se caracterizan como un "nuevo modo de regulación" diferente al libre mercado y al modelo burocrático tradicional. El casi-mercado es un "modo de regulación que preserva el financiamiento público pero incorporando mecanismos de mercado, como la libre elección y la competencia entre establecimientos, a través de una fórmula de financiamiento a la demanda" (Vanderberghe, 2000, p. 22). Por ello se sostiene que son objeto de un doble control: aquel de la administración pública y aquel de los clientes o usuarios que son libres de ir y venir, lo que condiciona la sobrevivencia de la escuela. En este sentido encuentran correspondencia con el modelo de los vouchers educativos basado en la teoría de la elección pública o public choice (Cosse, 1999).

Levic (1995) ${ }^{5}$ caracteriza al casi-mercado, distinguiéndolo de un servicio público, por la separación entre comprador y proveedor, la provisión del servicio se separa de su financiación de manera que distintos proveedores pueden competir por la prestación del servicio y la elección está a cargo del usuario. El casi-mercado está muy reglamentado, el gobierno mantiene el control de cuestiones tales como la entrada de nuevos proveedores, la inversión, la calidad del servicio y el precio que para el usuario generalmente es cero. Es decir, se combinan aspectos de un sistema intervencionista con un sistema de mercado: el Estado transfiere la responsabilidad directa de la educación y al mismo tiempo controla la provisión de los bienes sociales evaluando la calidad (Estado evaluador).

Es en este sentido que en el Proyecto de Ley de las Escuelas 2001 se establecía que "la reinvención de la escuela pública implica a la vez la reinvención del Estado educador". En el año 2002 la gobernadora de la Provincia de San Luis, María Luisa Lemme, expresó que el proyecto de las Escuelas Autogestionadas significa "una descentrali- 
zación muy importante en la educación, donde el rol del Estado pasa a ser el de un control muy fuerte y continuo". ${ }^{6}$

Las reformas educativas enmarcadas en esta lógica de casi-mercado educativo responden a una visión que pone el énfasis en las virtudes de lo privado y en la construcción de una nueva cultura institucional basada en las nuevas formas de gestión provenientes del ámbito empresarial, como respuesta a la crisis de los modelos tradicionales de gestionar la educación pública.

\section{Las Escuelas Autogestionadas en el contexto de la reforma}

El Proyecto de las Escuelas Experimentales o Autogestionadas de la Provincia de San Luis adquiere sentido en el marco de la denominada Transformación Educativa y de las políticas desarrolladas en las últimas décadas en Argentina, que, al igual que en gran parte de los países latinoamericanos, han estado orientadas por discursos y prácticas tendientes a legitimar un nuevo modelo económico, social, político, cultural y educativo.

La reforma educativa argentina de la década del ' 90 puede analizarse como una respuesta frente a la crisis de la matriz estado-céntrica que caracterizó la institucionalización y expansión del sistema educativo. La política educativa que acompañó el desarrollo del modelo del Estado de Bienestar Keynesiano, con las especificidades que este asumió en Argentina, de aspiración universalista y, según la legislación, con derechos accesibles para todos los ciudadanos, experimentó una reorientación que redefinió el carácter público de la educación y en consecuencia el papel del Estado como garante del derecho a la educación. Como consecuencia de este proceso comienza a redefinirse el sentido histórico de la educación pública vinculado con un modelo que, aún cuando no logró plasmar en la realidad, estuvo orientado por la utopía de la educación como un derecho de ciudadanía al servicio de un proyecto de movilidad e integración social. En el marco de la descentralización de los servicios sociales, la responsabilidad por la educación se traslada principalmente a las instituciones y a los individuos (Feldfeber, 2002).

Estas propuestas se inscriben en lo que Ball (2002) caracterizó como la "nueva ortodoxia" que redefine las relaciones entre la política, los gobiernos y la educación, y que está compuesta por diversos elementos que conforman un "nuevo consenso" que pretende conver- 
tirse en hegemónico. En primer lugar la ideología de mercado y las nuevas economías institucionales que explican el funcionamiento de la sociedad y sus instituciones en términos de las elecciones y acciones de un actor racional y se traducen en propuestas de autogestión local y mejoramiento escolar. La libre elección constituye otro de los elementos centrales en la conformación de este consenso. Por último se incluyen nuevos mecanismos de dirección y gestión que reemplazan a la intervención y a la prescripción por la fijación de objetivos y mecanismos de rendición de cuentas. Este nuevo tipo de gestión consiste en la apropiación de teorías y técnicas de la administración de empresas y del "culto a la excelencia" en las instituciones del sector público.

No puede entenderse la propuesta de las Escuelas Autogestionadas fuera del contexto en el cual se implementan. Por un lado, el proceso de reforma del Estado en el ámbito provincial en sintonía con el proceso de reforma en nivel nacional. Por otro, la implementación de propuestas de reforma del sistema educativo provincial tendientes a superar lo que considera el gobierno como "rigideces propias de un sistema centralizado y sobreregulado"7 que redefinen la relación entre el Estado y la sociedad. A su vez es necesario considerar las formas de construcción política instaladas en la provincia a partir de la Gobernación de Adolfo Rodríguez Saa, cuyo dominio en la provincia es absoluto, y el imperio que construyó en 17 años de gestión, un caso sin precedentes en la historia argentina, parece inamovible. ${ }^{8}$

Es en este marco que la reforma educativa, en la Provincia de San Luis, sustentada en la Ley de Educación de la Provincia n ${ }^{\circ}$ 4.947/ 92 del año 1992, estableció que la educación es responsabilidad de la familia, la sociedad y el Estado. Esta ley, sancionada un año antes que la Ley Federal de Educación (1993), extiende la denominación de escuela pública a todos los establecimientos, diferenciando las escuelas de gestión estatal y de gestión privada; conceptualización que retoma la Ley Federal de Educación. ${ }^{9}$

La Ley Provincial además establece la obligatoriedad en los niveles inicial y primario, sin estipular en ningún artículo la gratuidad; afirma el derecho a la libre elección de la familia del establecimiento educativo para sus hijos; señala que la igualdad de oportunidades sólo podrá ser garantizada con el aporte de distintos sectores de la comunidad; y en el capítulo referido al financiamiento, incluye dos artículos que apuntan al financiamiento compartido e incluso al autofinanciamiento (Pelayes, 2003). 
La Provincia también sancionó una serie de normativas que fueron redefiniendo el rol del Estado y de la sociedad en materia educativa a la vez centralizando las decisiones en el Poder Ejecutivo. Se cerraron instituciones, se crearon otras nuevas, se modificaron aspectos vinculados con las condiciones laborales de los docentes y se realizaron concursos para supervisores y directores por fuera de los mecanismos tradicionales de ascenso. Los procedimientos de estos concursos generaron mucha desconfianza porque no se difundieron en forma clara y pública los criterios para el otorgamiento del puntaje, los concursos no fueron públicos y el jurado fue designado por el Poder Ejecutivo (Pelayes, 2003).

También se creó un Fondo Educativo por Establecimiento Escolar de Gestión Estatal destinado a cubrir gastos para suplencias docentes, proyectos especiales; gastos de consumo, servicios y equipamiento; y gastos que promuevan la capacitación y la profesionalización docente.

\section{Las Escuelas A utogestionadas en la Provincia de San Luis: cuando la participación se define por decreto}

Las Escuelas Experimentales o Autogestionadas surgen por un acuerdo entre el Ejecutivo y una fundación que armó y vendió el proyecto a la Provincia. ${ }^{10} \mathrm{D}$ ebido a las resistencias en la comunidad educativa y las disidencias al interior del congreso, ${ }^{11}$ el Poder Ejecutivo no avanzó con su proyecto de ley y decidió imponer la reforma por decreto introduciendo algunas modificaciones con respecto a la propuesta original, entre ellas que no se convertirían escuelas "públicas tradicionales" en autogestionadas sino que se crearían nuevas escuelas. En el año 1999, el Decreto o $2.682^{12}$ estableció el llamado a convocatoria pública de proyectos educativos para la creación de nueve Escuelas Experimentales. Integraron el jurado evaluador miembros de la fundación involucrada en el proyecto. De este modo la autogestión que estas escuelas suponen surge como una política desde el Estado bajo la lógica de las reformas top-down 0 impuestas desde arriba.

El proyecto retoma el modelo de las charter schools (escuelas charter) de Estados Unidos. Las escuelas charter se definen como un híbrido de las escuelas públicas y las privadas. Son escuelas de inscripción abierta, financiadas por el Estado en base a la cantidad de alumnos, que funcionan en forma independiente y autónoma. Los alumnos, padres y maestros optan por participar en ellas. El gobierno, a través 
de un concurso, le otorga una licencia de funcionamiento a una asociación que dirige la escuela por un número determinado de años. El Estado evalúa y audita la marcha de la institución. Las escuelas compiten entre sí a través de sus proyectos.

Las Escuelas Autogestionadas en San Luis retoman con ciertas diferencias el modelo de las escuelas charter estadounidenses. Esas diferencias tienen más que ver con la forma de implementación que con el modelo que sustenta y orienta esas escuelas.

Las escuelas son gestionadas por Asociaciones Educacionales (asociaciones civiles sin fines de lucro) cuya selección se realizará a través de convocatorias públicas a cargo del Ministerio de Gobierno y Educación para la presentación de proyectos. Esas asociaciones deben estar integradas por no menos de tres docentes, pedagogos o personas cuyo perfil sea útil para los fines de la Asociación Educacional, y que en general tengan antecedentes relacionados con la educación. Tienen a su cargo la responsabilidad de organizar el proyecto pedagógico y la gestión de la institución, siendo además responsables por los resultados pedagógicos obtenidos, así como por la administración financiera de la institución. El decreto establece que las Asociaciones Educacionales "deberán ser democráticas y participativas, no podrán tener adscripción en especial y el proyecto educativo deberá respetar los principios de la educación pública argentina".

Se plantea que las Asociaciones Educacionales deberán "proveer una educación de calidad en los establecimientos que administren, a partir del logro de aumentos sostenidos en los aprendizajes y competencias de los alumnos". Para eso, deben elaborar un Proyecto Educativo Institucional (PEI), con el fin de proponer las líneas de acción que llevarán adelante, y los objetivos concretos a alcanzar. El PEI es el instrumento principal que el Ministerio de Gobierno y Educación utiliza para supervisar el logro de los estándares planteados para el aprendizaje de los alumnos.

En el decreto se plantea que el PEI deberá abarcar cada uno de los ámbitos de organización y decisión de la institución educativa, como la estructura de gobierno propuesta para la Asociación Educacional, la forma de organización financiero-contable, el plan de uso de los recursos que se planea llevar a cabo y, en particular, la organización pedagógica propuesta para la escuela experimental. También se señala que deberá ser claro y específico en lo que se refiere a las metas de aprendizaje a alcanzar, los mecanismos de información, internos y externos, que se estable- 
cen sobre el desempeño pedagógico, y con respecto a las innovaciones didácticas y pedagógicas que se implementarán con el fin de lograr una mejor calidad educativa.

El Comité Evaluador es quien evalúa los Proyectos Educativos Institucionales, los antecedentes de los miembros de las Asociaciones Educacionales, la defensa que realizan de sus proyectos y elabora una terna de acuerdo con un orden de mérito de las Asociaciones Educacionales postulantes para cada escuela, estableciendo los fundamentos de la elección. ${ }^{13}$

La Asociación Educacional cuyo proyecto es seleccionado por el Comité Evaluador obtiene una autorización de funcionamiento por un período de cinco años, al término del cual deberá participar en un nuevo concurso para obtener una renovación. El decreto posibilita en casos excepcionales a autorizar a una misma Asociación Educacional a administrar más de una escuela experimental. Se considera causal de revocación de la autorización la continuidad de resultados insatisfactorios en el desempeño de los alumnos, así como el uso indebido de los fondos recibidos.

El financiamiento está a cargo del Estado a través de un sistema de asignación por alumno o Unidad de Subvención Escolar (USE). Las escuelas no pueden establecer ningún tipo de arancel. La uSE es entregada mensualmente a las Asociaciones Educacionales, se calcula a partir de un Monto Base Mensual (MBM) de cien pesos (\$ 100) y varia de acuerdo al nivel educativo, a la zona en la cual funciona el establecimiento, ${ }^{14} \mathrm{y}$ a la provisión o no del edificio por parte del Estado Provincial.

D e la totalidad de los fondos que recibe la Asociación Educacional, puede invertir en salarios del personal hasta un 85\%. El resto debe invertirse en capacitación, equipamiento, bienes de consumo y otras erogaciones que se consideren necesarias para el mejor funcionamiento de la escuela.

En lo que respecta al Régimen Laboral Docente, el decreto establece que los docentes que optan por trabajar en esta escuelas dejan de depender del Estado y de estar amparados por el estatuto del docente para convertirse en empleados de las Asociaciones Educacionales, que gozan de libertad para establecer el régimen laboral y los salarios que no podrán ser inferiores, ni superiores en más del 50\%, del resto de las escuelas públicas. El personal que cumpla dos años de trabajo continuo en funciones de docencia tiene derecho a incorporarse como asociado a la Asociación Educacional. 
Las Asociaciones Educacionales firman un "Compromiso de Escuela de Calidad" y deben realizar, al menos una vez al año, una auditoría completa de sus estados financieros, legales y contables, para la cual deben contratar organismos y/ o profesionales independientes. Los resultados deben ponerse a disposición de los asociados, personal de la institución y miembros de la comunidad de la escuela.

Las Asociaciones Educacionales no pueden establecer políticas de discriminación al ingreso o permanencia de los alumnos. En el caso de que la cantidad de aspirantes que pretendan ingresar a una escuela experimental sea mayor que el espacio disponible para albergarlos, la Asociación Educacional deberá aplicar, junto con los criterios de ingreso vigentes en el resto de las escuelas públicas de la provincia, métodos de selección al azar que garanticen la igualdad de oportunidades en el ingreso de los aspirantes.

El decreto establece que es responsabilidad del Ministerio de Gobierno y Educación organizar un sistema de apoyo y orientación para las Escuelas Experimentales, con el fin de lograr un máximo aprovechamiento de las posibilidades que brinda ese sistema.

Al igual que la sanción de la Ley Provincial y las medidas implementadas en los últimos años no fueron fruto del consenso de la comunidad, las Escuelas Experimentales no fueron una demanda de la comunidad educativa que, muy por el contrario, expresó y expresa en buena medida su rechazo a la propuesta por considerarla una forma de privatización de la educación pública y de introducción de los mecanismos y valores de mercado. ${ }^{15}$ La autonomía que esas escuelas proponen surgió como una imposición del gobierno más que de una construcción desde la base. Es por ello que una directora de una de las Escuelas Autogestionadas expresó: "Nosotros no somos bien vistos por el resto de la comunidad educativa".

\section{Las Escuelas Autogestionadas: del texto del decreto a su contexto de implementación}

\section{L a autonomía al servicio de la innovación y la calidad}

En los fundamentos del Decreto del Régimen de Escuelas Experimentales, se establece como objetivo de la reforma lograr la "autonomía con el fin de promover la innovación y la calidad educativa".

La "libertad para crear" y formular el propio proyecto y la posibilidad de innovar son señaladas como las características centrales de estas 
escuelas, tanto por los técnicos de las fundaciones como de los directivos que integran las asociaciones que dirigen los Proyectos Educativos Institucionales. Así lo expresa una de las directoras: "Una ventaja es evidente que poder elaborar un proyecto y llevarlo adelante y tener la capacidad económica de solventarlo es realmente fascinante" (directora de una Escuela Autogestionada). Por eso señalan que lo que distingue a las Escuelas Autogestionadas es "la apertura al cambio".

La innovación se presenta asociada a la forma de gestión institucional. La posibilidad de elegir a los docentes y manejar los recursos para implementar el proyecto constituyen las características centrales de un nuevo modelo de gestión institucional.

A la hora de describir las innovaciones que estas nuevas formas de gestión introducen en las experiencias cotidianas de los alumnos, se señala que el trabajo en talleres y las actividades de apoyo escolar constituyen los ejes innovadores de estas instituciones. "Lo que estas instituciones identifican como innovaciones son estrategias que sientan sus bases en el modelo de trabajo por talleres, con nombres diferentes tales como 'laboratorios', 'bandas'. En ellos se intenta poner en acción lo trabajado en el currículo prescripto más los intereses de los alumnos puestos sobre determinados conocimientos" (Gobierno de San Luis y CIPPEC, 2002, p. 18).

Sin embargo es necesario señalar que estas formas de trabajo constituyen experiencias que ya han sido desarrolladas, con diversos grados de profundidad y sistematicidad, tanto por algunas escuelas públicas "tradicionales" como por escuelas privadas en diversas jurisdicciones del país. Lo que si constituye una diferencia relevante es que las Escuelas Autogestionadas tienen más horas de clase que las escuelas públicas tradicionales de la provincia.

Las Asociaciones Educacionales firman un "Compromiso de Escuela de Calidad" con la comunidad educativa y con el Ministerio de Gobiemo y Educación que constituye un aspecto central para evaluar el desempeño de cada Asociación Educacional. El contrato de adjudicación de las Escuelas Autogestionadas especificaba que los resultados obtenidos por esas escuelas en la evaluación del Sistema Nacional de Evaluación de la Calidad (SINEC) no podían ser menores que la media provincial. Esta meta no se cumplió en el año 2000. ${ }^{16}$ Si bien es entendible que, habiendo transcurrido sólo un año de implementación de la propuesta no se modificaran los contratos de adjudicación, esto pone en evidencia que la aplicación de las normas de manera flexible puede significar un alto grado de discrecionalidad, teniendo en cuenta las formas históricas de manejo político en la Provincia. ${ }^{17}$ 
En cuanto a la auditoría de las Escuelas Autogestionadas, la misma estuvo hasta fines del año 2002 a cargo de la Fundación CIPPEC integrada por algunos de los técnicos que desde la Fundación Gobierno y Sociedad que diseñaron el proyecto y lo ofrecieron a la Provincia. ${ }^{18}$ En este sentido se puede poner en cuestión la "supuesta objetividad" de la evaluación externa por parte de quienes son parte "interesada" en el éxito de la experiencia.

En lo que respecta al grado de autonomía con el que se manejan las escuelas se pueden realizar, de acuerdo con los testimonios recogidos, varias observaciones. En primer lugar, que el discurso oficial sobre esas escuelas homologa autogestión con autonomía, al mismo tiempo que innovación se presenta como sinónimo de mejoramiento de la calidad, supuestos que deben ser puestos en cuestión en función de la experiencia desarrollada hasta la fecha.

En segundo lugar la autonomía de los docentes se ve limitada en tanto no todos puedan participar efectivamente en las decisiones de las Asociaciones Educacionales y la definición de los proyectos que orientan el trabajo de las escuelas. Es por ello que cabe preguntarnos, de acuerdo con Contreras (1997), si la apelación a la autonomía de los docentes constituye un elemento para potenciar su trabajo profesional o sólo constituye una estrategia oficial para hacerle creer al docente que posee las condiciones de trabajo y es el único responsable por los resultados que obtienen los alumnos.

En tercer lugar, los valores vinculados con la democratización social no aparecen como centro de esta propuesta, o en todo caso, cuando se incorporan, se presentan asociados al discurso eficientista sobre la gestión y los nuevos modos de gerenciamiento más cercanos al mundo empresarial que al campo político orientado a garantizar la materialización de los derechos de los ciudadanos.

Además, si bien las escuelas cuentan con autonomía para manejar los fondos, el financiamiento basado en la cantidad de alumnos puede convertirse en un factor que atente contra la calidad en tanto en la permanecia de los alumnos se juega la supervivencia de la institución. Hasta el momento no hay competencia entre las escuelas por captación de matrícula debido a la reducida cantidad de instituciones. No obstante, la lógica de la competencia está presente en la experiencia.

Por un lado, al interior de algunas de las escuelas el sector docente se ve sometido a competir por obtener mejor salario atado a los resultados de los alumnos y a la evaluación que de su tarea realiza la 
desde que se implementó esta experiencia, estas instituciones están constantemente en el centro de la atención, lo que lleva a directivos y docentes a una sensación de constante necesidad de mostrar resultados e innovaciones. D e este modo, la competencia no se da entre las escuelas autogestionadas, sino más bien del conjunto de las autogestionadas en relación con las escuelas del sistema público. Sin embargo, es necesario tener en cuenta que la ausencia de competencia entre las escuelas autogestionadas se debe a lo limitado de la experiencia, y no a características intrínsecas, dado que el presupuesto se les otorga a las escuelas sobre la base de una subvención por alumno y no hay limitación alguna en la norma respecto de cuántas escuelas pueden abrirse. (Jaimovich y Saforcada, 2003, p. 13)

\section{L as condiciones de trabajo: la flex ibilización laboral de los docentes}

Las condiciones de trabajo de los docentes constituyen un aspecto central en el funcionamiento de esas escuelas. ${ }^{19}$ Por un lado se valora el hecho de que las Asociaciones Educacionales pueden seleccionar a los docentes de modo de poder conformar sus equipos de trabajo. Tanto para el discurso oficial como para los miembros de esas asociaciones poder conformar el equipo docente constituye un elemento central para llevar adelante una experiencia innovadora, diferenciándose de este modo de las escuelas públicas tradicionales donde la conducción del establecimiento no puede elegir a los docentes que van a desempeñarse en la escuela. Sin embargo estas condiciones también se han visto limitadas en la medida de que en general son los docentes más jóvenes, sin experiencia y con menos antigüedad quienes se han presentado para trabajar en esas escuelas, debido a la resistencia que el proyecto generó entre los docentes en general y en especial entre aquellos de mayor antigüedad en la docencia. Los sindicatos docentes desempeñaron un papel clave en la oposición a las Escuelas Autogestionadas por considerar que atentan contra los derechos de los docentes como trabajadores y porque significan una forma de privatización de la educación pública debido a que con fondos públicos se financian escuelas gestionadas por particulares.

Por otro lado, la inestabilidad laboral constituye una condición que genera mucha inseguridad entre los docentes; hecho que atenta contra la construcción de un proyecto compartido y sostenido a largo plazo. Este hecho es reconocido tanto por los técnicos del CIPPEC como por los docentes que trabajan en esas escuelas. "La percepción de inestabilidad se convierte en un obstáculo para alcanzar el mejor compro- 
miso con el trabajo institucional" (Gobierno de San Luis y CIPPEC, 2002, p. 22).

También el hecho de que los docentes perciban en algunas escuelas salarios diferentes ha generado situaciones conflictivas. No están claros los criterios de asignación de los premios y bonificaciones que establecen diferencias en la escala salarial y a veces hay criterios contrapuestos o diferentes entre los miembros de la Asociación Educacional y los directores (aún cuando estos también integren la asociación). ${ }^{20}$

En función del establecimiento de criterios salariales que diferencien el trabajo de los docentes premiando al que "trabaja bien" y castigando al que "trabaja mal", surge el interrogante sobre las posibilidades efectivas para la construcción colegiada de un proyecto articulado en torno a objetivos comunes. Es decir, cabe preguntarse si es posible pensar en un proyecto democratizador cuando los valores que se promueven son la meritocracia y la competencia por lograr mejores salarios.

\section{5. ¿Son públicas las Escuelas A utogestionadas?}

Las Escuelas Autogestionadas son caracterizadas tanto desde el discurso oficial como desde los técnicos involucrados en el proyecto como escuelas públicas debido a que son gratuitas, están financiadas por el Estado y son de libre acceso para los alumnos. Más aún, consideran que estas escuelas refuerzan el carácter público de las escuelas tradicionales en tanto tienen libertad para definir un proyecto adaptado a las necesidades de los alumnos y a las demandas de la comunidad, lo que redunda en una mayor participación de los actores involucrados. Los docentes de las Escuelas Autogestionadas si bien reconocen que son escuelas públicas, a la hora de caracterizarlas lo hacen en términos de "escuelas públicas de gestión privada". ${ }^{21}$

Sin embargo debemos señalar que son los intereses propios de la esfera privada los que llevan a los particulares a conformar una Asociación y participar de la licitación de los proyectos. De acuerdo con un informe elaborado por el Gobierno de la Provincia y el CIPPEC (2002), es la "sociedad civil que se hace responsable" a través de la participación en las iniciativas públicas de personas y entidades que tienen "interés legítimo". Al respecto cabe preguntarnos quién define cuál es el interés legítimo y en qué se basa y cómo se construye dicha legitimidad. En el mismo informe se define en forma contradictoria 
la responsabilidad del Estado: "El Estado sin abandonar la responsabilidad a lo que demanda el sistema educativo transfiere responsabilidades, atribuciones y los recursos a las asociaciones educacionales".

En torno a las Escuelas Autogestionadas se articulan los intereses de diversos grupos: por un lado de la fundación que arma y le vende el proyecto a la Provincia (Fundación Gobierno y Sociedad), algunos de cuyos miembros integran la segunda fundación (CIPPEC), que audita la marcha del proyecto. Por otro los intereses de la Provincia en hacer más eficiente la gestión del sector público, transformar las escuelas en organizaciones que funcionen como empresas y modificar la carrera de los docentes de modo de quebrar la lógica de accionar del sindicalismo docente que constituye uno de los sectores de oposición al Gobierno Provincial. Por último los intereses de los particulares que conforman la Asociación Educacional y se presentan a la Convocatoria Pública para la selección de proyectos. ${ }^{22}$

De acuerdo con algunos de los testimonios de los docentes, las Escuelas Autogestionadas "son públicas para los alumnos y privadas para los docentes", públicas para los alumnos en tanto no establecen requisitos de acceso y son gratuitas; privadas para los docentes porque el régimen de contratación y la inestabilidad laboral son los que rigen para el sector privado.

Con respecto a la participación de los actores - que constituye un aspecto central para definir el carácter público de estas escuelas es necesario realizar varias observaciones. En primer lugar el proyecto es elaborado por la Asociación Educacional que luego de ganar la licitación contrata a los docentes, por lo tanto estos en su gran mayoría no han participado de la definición inicial de la propuesta.

En segundo lugar la participación de los docentes en la Asociación Educacional a partir de los dos años de desempeño en la escuela, tal como está previsto en el decreto, se ha encontrado con obstáculos en la práctica, ya que en algunos casos los miembros de las asociaciones decidieron interrumpir los contratos de los docentes una vez cumplidos los dos años, de modo tal de no tener que incorporarlos a la asociación.

En lo que respecta a la participación de los padres se observa en función del relato que realizan sobre las actividades en las que participan los padres tanto en las encuestas como en las entrevistas - que hasta el momento no han logrado construirse vínculos entre las escuelas y las familias diferentes a los que se establecen en las escuelas públicas tradicionales. ${ }^{23}$ 
En tanto la participación escolar es necesariamente política, ella está relacionada con la reconstrucción democrática y con la constitución de identidades ciudadanas y con la educación misma (Beltrán Llavador, 1998). D esde esta perspectiva, podemos señalar que el modelo de participación que subyace al modelo de las Escuelas Autogestionadas se vincula más con una racionalidad económica de corte eficientista y en el paradigma de la accountability o rendición de cuentas que con una propuesta de reconstrucción democrática en el contexto del sistema educativo de la Provincia de San Luis.

Acerca de la libertad de elección de los padres, que constituye uno de los fundamentos que sustentan el modelo de esas escuelas, hay que señalar que en general las Escuelas Autogestionadas se abrieron en zonas donde faltaban vacantes, por lo tanto los padres concurrieron a esas escuelas principalmente porque encontraron vacantes para enviar a sus hijos y porque quedan cerca de sus hogares.

Algunas de las escuelas se han ubicado en zonas marginales y atienden a población en condición de pobreza, lo que es señalado por los funcionarios y los expertos como un componente de equidad y justicia social de la propuesta. Sin embargo cabría preguntarnos si este objetivo no podría lograrse con la apertura de escuelas públicas tradicionales. No queda claro por qué la autogestión que esas escuelas proponen favorecería a los sectores más pobres o por el contrario podría reproducir 0 agudizar aún más las diferencias - ya existentes - entre las escuelas en función de la población que atienden.

$\mathrm{Si}$ definimos a las Escuelas Autogestionadas como organizaciones públicas no-estatales, es decir si entendemos a la sociedad civil como el conjunto de organizaciones no-gubernamentales ni burocrático-estatales ni mercantiles, de acuerdo con Fraser (1994), la distinción entre sociedad civil y Estado daría como resultado la existencia de "públicos débiles", ya que esa esfera de los públicos débiles promovería la deliberación, y formaría opinión pero estaría separada de los órganos del Estado donde se toman las decisiones en nombre del bien común. En cambio los poderes legislativos y ejecutivos funcionarían como "públicos fuertes", públicos cuyo discurso abarca la formación de opinión y la toma de decisiones. Desde esta perspectiva podríamos entender el lugar del Gobierno Provincial y de las fundaciones involucradas en el proyecto como públicos fuertes que logran hacer oir su voz y tomar decisiones que plasman en legislaciones "fuertes", y a los docentes y la comunidad de cada una de las escuelas como públicos débiles, que pueden gestionar sus proyectos sujetos a los avatares de los acuerdos en 
nivel central. La construcción del espacio público que esas escuelas suponen está centrada en el espacio "individual" de cada institución que se transforma en la responsable por los resultados obtenidos.

\section{Reflexiones finales}

El Proyecto de las Escuelas Autogestionadas de San Luis adquiere sentido en función de la coyuntura en la que se implementa y de la forma en la cual se impone. No constituye un proyecto que responda a las reivindicaciones de la sociedad sino a una delegación de responsabilidades por parte del Estado a partir de una propuesta diseñada por técnicos que importan el modelo de las escuelas charter estadounidenses.

Juntos a una retórica basada en la eficiencia en la gestión, se retoman reivindicaciones históricas en torno a la autonomía y la participación para dotarlas de un nuevo sentido a la par que redefine el imaginario histórico que la educación pública asumió en Argentina y consecuentemente el papel asignado al Estado como garante del derecho a la educación como derecho de ciudadanía.

El sentido de lo público se redefine en un doble proceso que estira sus límites para incorporar a las formas de gestión privadas o particulares dentro de las formas públicas no estatales, a la par que restringe el carácter público a la gratuidad del servicio, al libre acceso para todos los alumnos y a la responsabilidad por los resultados obtenidos en función de la gestión autónoma del proyecto. Los valores vinculados con la democratización social, la igualdad, la justicia, el bien común y la solidaridad no se presentan en la discusión acerca del carácter público de esas escuelas. Tampoco se incluye una mirada integral acerca de la escuela en el marco del sistema educativo, reduciéndose el problema a la búsqueda de calidad de cada escuela individual, hecho refuerza la segmentación del sistema. La discusión política acerca del sentido de la reforma queda soslayada bajo la supuesta neutralidad del modelo que orienta la propuesta.

La autonomía y la emancipación no pueden lograrse desde la imposición tecnocrática de modelos que hasta la fecha han contribuido a agudizar las desigualdades educativas y sociales y han significado formas de privatización del espacio público de la escuela.

Recebido em julho de 2003 e aprovado em agosto de 2003. 


\section{Notas}

1. Al respecto ver entre otros: Asociacion Cristiana de Dirigentes de Empresas (ACDE). Propuesta de transformación en la gestión de la educación en la A rgentina, Comisión de Educación, Buenos Aires, 2000; Blanco Etchegaray et al. L a escuela protagonista. Grupo Sophia, Temas Grupo Editor, Buenos Aires, 1999; Campora et al. H acia una escuela con mayor autonomía, Grupo Sophia, Fundación Banco de Boston, Buenos Aires, 1996; William Experton. D esafíos para la nueva etapa de la reforma educativa en A rgentina, Informe del Banco Mundial, 1999; Fundacion de Investigaciones Economicas Latinoamericanas (FIEL). U na educación para el siglo X X I. Propuesta de reforma, Centro de Estudios Públicos, Buenos Aires, 2000; G rupo Sophia. A utonomía escolar. ¿Q ué opinan los docentes?. Fundación Banco de Boston, Buenos Aires, 1998; Llach, J.J. et al. E ducación para todos, Instituto de Estudios sobre la Realidad Argentina y Latinoamericana (IERAL), Fundación Mediterránea, Córdoba, 1999; E. Vegas; L. Pritchett y W. Experton. Cómo atraer y retener docentes calificados en la A rgentina: impacto del nivel y estructura de la remuneración, Human Development Department, Banco Mundial, 1999.

2. Fundamentos del Proyecto de Ley de Creación del Sistema de Escuelas 2001, San Luis, abril de 1999.

3. El trabajo se inscribe en el Proyecto de Investigación UвACYт "Privatización y reforma educativa. La experiencia argentina de la década del noventa". Instituto de Investigaciones en Ciencias de la Educación, Facultad de Filosofía y Letras, Universidad de Buenos Aires. Directora: Myriam Feldfeber. El trabajo de campo sobre las Escuelas Experimentales en San Luis estuvo a cargo de Analía Jaimovich y Fernanda Saforcada.

4. El trabajo de investigación incluyó, además del relevamiento documental, entrevistas a los responsables de las fundaciones involucradas en el proyecto y a integrantes del equipo de auditoría y un trabajo de campo en la Localidad de Villa Mercedes en tres de las nueve Escuelas Autogestionadas que funcionan en la Provincia (cinco de las cuales comenzaron a funcionar en el año 2000 y quatro en el año 2001). Se realizaron entrevistas a los directores de cada una de las escuelas, a coordinadores y a docentes. Se aplicaron encuestas a docentes, padres y alumnos de los tres establecimientos. También se realizaron algunas entrevistas y encuestas en dos escuelas públicas de la localidad pero con gran dificultad debido al rechazo de los docentes a participar de cualquier acción que tenga relación alguna con el proyecto de Escuelas Autogestionadas, aún cuando la investigación se realiza en el marco de la universidad y no tiene vinculación alguna con el gobierno. También se entrevistaron a representantes del sindicato, de la Coordinadora de Padres y a un funcionario provincial.

5. Citado en Whitty et al. (1999).

6. D eclaraciones de la gobernadora de la Provincia de San Luis a raíz de las marchas en contra del Proyecto de Escuelas Autogestionadas y de los cambios en el Ministerio de Educación. Lunes, 2 de septiembre de 2002.

7. Fundamentos del Proyecto de Ley de Creación del Sistema de Escuelas 2001, San Luis, abril de 1999.

8. La periodista Olga Wornat lo describe en los siguientes términos: "Tiene un perfil similar al que tenía Menem. Es un caudillo provincial que logró un crecimiento macroeconómico, un orden fiscal y un desarrollo de infraestructura con alto impacto en el descenso de la población con necesidades básicas insatisfechas. Allí conviven el equilibrio macroeconómico y un fuerte desequilibrio en la distribución del ingreso". Rodríguez Saa es, desde hace 18 años, el amo y señor de San Luis, una provincia a la que llegó como gobernador en 1983 y a la que él denomina "El Paraíso" de la Argentina. Olga Wornat, El Adolfo de San Luis, en: Revista Poder, 27 de noviembre de 2002. 
9. La histórica distinción entre los ámbitos público y privado, en materia educativa, se reduce a un problema de gestión y la educación pública se redefine como "educación pública de gestión estatal". El concepto de "lo público" se amplía, incluyendo también a la educación privada que pasa a ser considerada como "educación pública de gestión privada". El supuesto que subyace a esta conceptualización es que toda la educación es pública, residiendo la diferenciación público-privado en la posesión del edificio y en la gestión del servicio.

10. El Cconvenio estableció la asistencia técnica a la Provincia por parte de la Fundación Gobierno y Sociedad para el diseño, ejecución y contralor del Proyecto "Escuelas 2001". El gobierno de la Provincia se comprometió a otorgar el equipamiento necesario para el desarrollo de las tareas y a abonar en 10diez cuotas mensuales la suma total de \$ 170.000 (en eseaquel momento equivalían a 170.000 dólares).

11. Aún cuando el partido gobernante contaba con mayoría parlamentaria, había también desacuerdos dentro del propio partido gobernante.

12. El decreto se basó en la Ley de Escuelas Experimentales nำ 4.914, sancionada en la Provincia en el año de 1991.

13. Pueden declarar desierta la convocatoria en caso de que se considere que las Asociaciones Educacionales y sus proyectos no reúnen los requisitos necesarios.

14. El Poder Ejecutivo determina a que zona corresponde cada una de las escuelas concursadas: urbanas o rurales y/ o de alto riesgo social.

15. La oposición está liderada principalmente por el gremio provincial AmPPYA (Agremiados del Magisterio Provincial Puntano y Afines) y la Coordinadora de Padres.

16. El informe de auditoría lo atribuye a que el proyecto sólo tenía un año de existencia y a que los alumnos provenían con diversas formaciones de instituciones públicas y privadas.

17. La discrecionalidad en la aplicación de las normas es un rasgo común a muchas provincias del país.

18. De acuerdo con el tipo de Asociación Educacional (nivel educativo que brinda 0 modalidad), se les descontaba un porcentaje del presupuesto que la escuela percibe para cubrir los costos de la auditoría. D esde el año 2003 no se efectúa esa retención porque la auditoria está a cargo del Ministerio de Educación de la Provincia.

19. Las propuestas de flexibilización laboral encuentran su correlato en las reformas impulsadas en nivel nacional para el conjunto de los trabajadores. Las propuestas del Banco Mundial para Argentina se inscriben en esta línea que propone derogar los Estatutos D ocentes y definir una nueva carrera docente basada en el mérito y en el establecimiento de incentivos para atraer a los personas más calificadas a la docencia.

20. En algunos casos estas diferencias obedecen a que los miembros de las Asociaciones Educacionales privilegian los aspectos más formales del trabajo, como la asistencia, y los directores se centran en cuestiones más vinculadas con el proceso pedagógico en el aula.

21. Del mismo modo las han caracterizado la mayoría de los padres encuestados en las tres Escuelas Autogestionadas donde se realizó el trabajo de campo.

22. Una de las Asociaciones Educacionales a cargo de una de las Escuelas Autogestionadas está integrada por miembros de una escuela privada de otra provincia ya que el decreto no establece que los miembros de esas asociaciones deben residir en la provincia aunque si se establece que debe realizarse un diagnóstico de la comunidad donde va a funcionar la escuela.

23. Es elocuente el testimonio de una de las directoras de las Escuelas Autogestionadas: “(...) en algunas actividades trabajamos conjuntamente con los padres, no en todas. Es un 
aprendizaje lento porque esta escuela fue una aspiración de muchos y los procesos de enseñanza/ aprendizaje nos gusta que la injerencia sea del docente. No quiere decir esto que los padres no estén a tono de la información que requieren porque nosotros trabajamos un cuaderno de comunicaciones gratuito pero obligatorio (...) el que no lo trae tiene alguna sanción o media falta, entonces se preocupan por traerlos. Y ahí se vuelca toda la información de las notas, de las faltas, de quién se comportó mal".

\section{Referencias bibliográficas}

BALL, S. Grandes políticas, un mundo pequeño: introducción a una perspectiva internacional en las políticas educativas. In: NAROD OWSKI, M.; Nores, M.; And RAdA, M. (Comp.). Nuevas tendencias en políticas educativa: Estado, mercado y escuela. Buenos Aires: Granica, 2002.

BELTRAN LLAVAD OR, F. La inevitable necesidad de la participación en la escuela (pública). Revista E nfoques E ducacionales, Chile, v. 1, n. 2, 1998.

BO URD IEU, P. C ontrafuegos: reflexiones para servir a la resistencia contra la invasión neoliberal. Barcelona: Anagrama, 1999.

BRESSER PEREIRA, L.C. La reforma del Estado de los años noventa: lógica y mecanismos de control. Revista D esarrollo E conómion, Buenos Aires, v. 38, n. 150, jul./ sep. 1998.

BRESSER PEREIRA, L.C.; CUNILL GRAU, N. L o público no-estatal en la reforma del E stado. Buenos Aires: Paidós, 1998.

CICCIONI, A. El movimiento de las charter schools: una amenaza y una oportunidad para la educación pública argentina. Revista Propuesta E ducativa, Buenos Aires, v. 10, n. 20, 1999.

CO NTRERAS, J. L a autonomía del profesorado. Madrid: Morata, 1997.

COSSE, G. El sistema de voucher educativo: una nueva y discutible panacea para América Latina. Revista Propuesta E ducativa, Buenos Aires, v. 10, n. 20, 1999.

FRASER, N. Reconsiderando la esfera pública: una contribución a la crítica de la democracia existente. Revista E ntrepasados, Buenos Aires, v. 4, n. 6, 1994.

GOBIERNO DE LA PROVINCIA DE SAN LUIS/ CIPPEC. Libertad para crear. de la autogestión escolar a la auditoría estatal. San Luis, CIPPEC, 2002. 
MUNIN, H. L a autonomía de la escuela: un recorrido por la discusión alemana de los '90. Buenos Aires: Aique, 1999.

NAROD OWSKI, M. Varias respuestas a diez objeciones efectuadas a las escuelas públicas autogestionadas: un aporte al debate en la Argentina. Buenos Aires: Fundación Gobierno y Sociedad, 1999. Disponível em: <escolares.com.ar/ paralaescuela/ utilesdocente/ debates ponencias/ escuelas_autogestionadas_narodowski.htm> Acesso em: sêt. 2003.

PELAYES, O. La autonomía escolar como ropaje de la privatización y recentralización educativa. In: MANG O, M.; VAZquez, S. E 1 debate del espacio público en la escuela. Buenos Aires: CTERA; SNES, 2003.

JAIMOVICH, A.; SAFO RCADA, F. Reforma educativa y redefinición de lo público: el caso de las escuelas autogestionadas de San Luis. In: COLOQUIO Nacional a diez años de la Ley Federal de Educación, jun. 2003, Cordoba. Cordoba: Universidad de Córdoba, 2003.

VANDERBERGHE, V. Análisis de la relación entre financiamiento de la demanda y la calidad y equidad de la educación. Revista de Tecnología E ducativa, Santiago de Chile, v. 4, n. 1-2, 2000.

WHITTY, G.; POWER, S.; HALPIN, D. La escuela, el E stado y el mercado: delegación de poderes y elección en educación. Madrid: Morata; Paideia, 1999. 